\title{
Reflexões sobre interdisciplinaridade e multidisciplinaridade na formação de professores em ambiente de m-learning
}

\author{
Valkiria Venancio $^{1,2}$, Irene Karaguilla Ficheman ${ }^{1}$, Roseli de Deus Lopes ${ }^{1}$ \\ ${ }^{1}$ Universidade de São Paulo (USP) - Escola Politécnica - Sistemas Eletrônicos - Núcleo de \\ Aprendizagem, Trabalho e Entretenimento do Laboratório de Sistemas Integráveis \\ Cep - 05508-010 - Cidade Universitária - São Paulo - SP - Brasil \\ ${ }^{2}$ Universidade de São Paulo (USP) - Faculdade de Educação - Departamento de Metodologia do \\ Ensino e Educação Comparada - Grupo de Estudos e Pesquisa em Etnomatemática \\ Cep - 05508-900 - Cidade Universitária - São Paulo - SP - Brasil \\ (venancio, irene, roseli)@lsi.usp.br
}

\begin{abstract}
This article describes three projects created and developed by educators in schools ProUCA during their training. Categorizing them as multidisciplinary or interdisciplinary based on concepts defined here. As projects developed in m-learning environment reflects on the assumptions of interaction, participation and access, technical and administrative assistance and management. The reflection is performed in order to begin rethinking the training of teachers in the learning environment using mobile technologies.

Resumo. Este artigo descreve três projetos criados e desenvolvidos por educadores de escolas do ProUCA durante a sua formação. Categoriza-os em multidisciplinares ou interdisciplinares tendo como base seus conceitos aqui definidos. Como projetos desenvolvidos em ambiente m-learning reflete-se sobre os pressupostos de interação, participação e acesso, questões técnicas e contribuição administrativa e gestora. A reflexão realizada tem o intuito de principiar o repensar da formação dos educadores nos ambiente de aprendizagem que utilizam tecnologias móveis.
\end{abstract}

Palavras chave: Multidisciplinaridade, interdisciplinaridade, formação de professores, m-learning 


\section{Introdução}

O Programa "Um Computador por Aluno" (ProUCA) do Ministério da Educação e Cultura (MEC), em sua segunda fase piloto, disponibilizou às mais de 300 escolas envolvidas, apoio e implantação técnica, acesso a Internet sem fio, formação semipresencial aos professores e gestores por meio de ambiente virtual, orientação para o uso de portais educacionais e objetos de aprendizagem. Desta maneira, houve em certo momento no ProUCA uma busca à constituição do ambiente de aprendizagem móvel (www.uca.gov.br).

A aprendizagem móvel ou mobile learning (m-learning), como modalidade de aprendizagem e de ensino em ambientes computacionais de aprendizagem voltados a treinamentos e ensino, surge com o crescente desenvolvimento de tecnologias móveis e da computação ubíqua [Al-Hmouz et al. 2009; Reis et al. 2012, in FREITAS e BARBOSA, 2012].

O m-learning é caracterizado pela sua capacidade de proporcionar forte interação entre os aprendizes e os instrutores, possibilitando a contribuição, participação e acesso ao ambiente de aprendizagem por meio de quaisquer dispositivos móveis a qualquer momento em qualquer lugar. Tais dispositivos utilizados nesta interação, por si só ou em combinação com outras tecnologias da informação e da comunicação (TIC), permitem uma aprendizagem desdobrada em vários formatos, assim como, apoia objetivos educacionais, administrativos ou de gestão [Laine et al. 2010, in FREITAS e BARBOSA, 2012; UNESCO, 2012]. Freitas e Barbosa (2012) apontam que o desenvolvimento de m-learning envolve diferentes fatores que devem considerar os aspectos técnicos, os componentes educacionais, atributos de computação ubíqua, critérios de usabilidade móvel, entre outros.

Na pesquisa de Santos e Borges (2011) se questiona a implantação de laptops educacionais no cotidiano escolar como promotores de mudanças curriculares ou somente reforço às estruturas existentes. Concorda-se que a presença única dos laptops na sala de aula certamente não promove uma educação inovadora. Para que isto realmente aconteça é imprescindível que professor, gestor, aluno e comunidade contribuam com as mudanças que estão por vir. Sem o empenho e o total envolvimento da escola estas mudanças não ocorrem.

A instituição escolar vê o laptop educacional como oportunidade de convergir pessoas e conhecimentos, integrando-os para que se completem e produzam aprendizagem, apesar de sentir grande dificuldade para incorporar estas inovações e avanços no cotidiano escolar (VENANCIO et al., 2012a).

Em uma série de pesquisas publicadas pela UNESCO (ISAACS, 2012; DERIQUITO e DOMINGO, 2012) demonstra-se que projetos de m-learning, na Ásia e África, com uso de telefones móveis e outras tecnologias, vem contribuindo para melhoria no desempenho escolar dos aprendizes em diversos níveis de ensino e em diferentes áreas do conhecimento.

A dinamicidade provocada pela integração da tecnologia em sala de aula, onde o professor oportuniza a participação ativa do aluno, incentiva sua autonomia e consequente expressão do pensamento crítico e formação de opinião ao atuar como protagonista da sua aprendizagem, além de agir como elemento de valorização do trabalho colaborativo e inter-relacional, somente ocorrerá com formação adequada e permanente de professores e gestores, que considere as realidades das escolas e seus projetos políticos pedagógicos, bem como as necessidades dos envolvidos, colocando o professor como foco da formação, em situações onde ele possa refletir sobre sua prática (BONA et al., 2011; VENANCIO et al., 2012a).

O uso das tecnologias educacionais móveis ainda depende de muitas variáveis alheias ao 
professor, gestor ou escola, mas cabe a eles transformar em aprendizagem eficaz o uso destes recursos e às políticas públicas estimular a adoção de tecnologias diversificadas, oferecer resoluções técnicas e formação permanente dos educadores (DANNEMANN, 2013).

Diante desta nova realidade, em São Paulo especificamente, observou-se em diferentes escolas participantes da formação do ProUCA, características próprias e individuais de organização e interação das pessoas com o uso de diversas tecnologias e dos laptop educacionais disponibilizados pelo programa (VENANCIO et al, 2012a; VENANCIO et al, 2012b).

Durante a formação e como parte das atividades desenvolvidas, diversos grupos de professores desenvolveram projetos colaborativos, que exigiram certa aproximação entre as disciplinas, com o intuito de se apoiarem mutuamente e apresentarem aos alunos temas significativos e de seu interesse.

Na seção 2 deste artigo, descreve-se objetivos da formação dos educadores do piloto do ProUCA. Na seção 3 apresenta-se a conceituação de multidisciplinaridade e interdisciplinaridade, descreve-se três projetos desenvolvidos durante a formação ProUCA 2011 e 2012 e os categoriza. $\mathrm{Na}$ seção 4 destacam-se as reflexões sobre os projetos sob os pressupostos do ambiente m-learning. E, finalmente, na seção 5 as considerações finais.

\section{Formação de educadores}

Os gastos com a formação de professores estão na proporção de 9:1 em relação aos gastos com a própria tecnologia (Blikstein, 2012 in DANNEMANN, 2013). Sem ela dificilmente o professor irá usá-las para ensinar de modo diferenciado.

Deste modo o MEC em parceria com as Universidades brasileiras e Secretarias Estaduais e Municipais de Educação de todo o Brasil responsabilizou-se em oferecer formação semipresencial para os professores e gestores das escolas ProUCA, por meio do E-proinfo - ambiente virtual de aprendizagem. O curso objetivou promover a integração do uso dos recursos tecnológicos em software livre ao currículo, na busca de qualidade educativa, usando a tecnologia como instrumento a favor do ensino e da aprendizagem.

Almeida e Prado (2011) afirmam e assim acrescentam que a formação docente para utilização do laptop educacional tem como pressupostos novas ações pedagógicas que apontem mudanças no currículo da escola, a promoção da inclusão digital e social e o reconhecimento da autonomia na organização curricular. Ainda, segundo as autoras, os professores são responsáveis pela dinâmica e inovação das aulas e os projetos na escola, por meio de práticas e utilização da tecnologia, que favoreçam a qualidade da aprendizagem dos estudantes. Silva e Petrucci (2012) complementam afirmando que a postura do professor incentivador da aprendizagem é fundamental para potencializar o processo. E, neste processo, os projetos colaborativos criados pelos professores e gestores no período de formação apontaram para uma perspectiva de mudança, já que "o bom uso das tecnologias para interação de modo cooperativo, colaborativo, favorecendo a autonomia $e$ tomada de decisões implica em assumir a responsabilidade pelas próprias escolhas" (FAGUNDES e JOST, 2013). 


\section{Projetos colaborativos}

Um dos conteúdos desenvolvidos na formação do ProUCA tratou especificamente da metodologia de ensino. Realizou-se reflexões sobre a Pedagogia Problematizadora, a Resolução de Problemas e a metodologia de Projetos de Aprendizagem. Na metodologia de projetos de aprendizagem, especificamente, o processo de investigação parte do tripé: questão principal, dúvidas temporárias e certezas provisórias, onde o grupo reunido por interesses comuns parte do conhecimento prévio para a validação, refutação ou aprofundamento destes conhecimentos.

Neste momento de estudos da formação dos professores, uma das atividades consistiu na elaboração e execução de projetos, o que provocou a aproximação de professores e/ou disciplinas sobre temas específicos.

O nível de aproximação entre as diferentes disciplinas na elaboração e execução de projetos aponta para estruturas multidisciplinares ou interdisciplinares.

\subsection{Multidisciplinaridade ou Interdisciplinaridade}

A multidisciplinaridade corresponde à busca da integração de conhecimentos por meio do estudo de um objeto de uma mesma e única disciplina ou por várias delas ao mesmo tempo. Este tipo de pesquisa traz contribuições significativas a uma disciplina específica, porque "ultrapassa as disciplinas, mas sua finalidade continua inscrita na estrutura da pesquisa disciplinar" (Nicolescu, 2000 in BICALHO e OLIVEIRA, 2011). Ou seja, as disciplinas estão perto, mas não juntas, estudam um determinado tema, sem a preocupação de interligar-se entre si.

Domingues (2005 in BICALHO e OLIVEIRA, 2011) destaca as principais características de experiências multidisciplinares:

- Aproximação de diferentes disciplinas para a solução de problemas específicos;

- Diversidade de metodologias, cada disciplina permanece com a sua metodologia;

- Os campos disciplinares, embora cooperem, guardam suas fronteiras e não se contagiam ao contato.

A interdisciplinaridade é uma interação entre duas ou mais disciplinas, acrescentando que "um grupo interdisciplinar compõe-se de pessoas que receberam formação nos diferentes domínios do conhecimento (disciplinas), tendo cada um conceitos, métodos, dados e temas próprios" (Berger, 1972 in BICALHO e OLIVEIRA, 2011). Também Jean Piaget (1972 in BICALHO e OLIVEIRA, 2011) acrescenta que na interdisciplinaridade "há cooperação e intercâmbios reais, enriquecimento mútuo". Tem como objetivo superar o isolacionismo e a independência das disciplinas, como forma de inovar a formação sócio-profissional e de superar a distância (Japiassu, 1976 in BICALHO e OLIVEIRA, 2011). A interdisciplinaridade "diz respeito à transferência de métodos de uma disciplina para outra" (Nicolescu et al., 2000 in BICALHO e OLIVEIRA, 2011).

Para Frigotto (1995) a interdisciplinaridade é "uma necessidade relacionada à realidade concreta, histórica e cultural, constituindo-se assim como um problema ético-político, econômico, cultural e epistemológico". Para tal, "é necessário uma coordenação para integrar os objetivos, 
atividades, procedimentos, atitudes, planejamentos e que proporcione o intercâmbio, a troca, o diálogo, etc". (NOGUEIRA, 2001).

Domingues (2005 in BICALHO e OLIVEIRA, 2011) destaca as principais características de experiências interdisciplinares:

- Aproximação de campos disciplinares diferentes para a solução de problemas específicos que envolvam uma realidade concreta;

- Compartilhamento de metodologia;

- Geração de novas disciplinas após cooperação e fusão entre os campos.

Para o desenvolvimento do conteúdo e atividades sobre Projetos de Aprendizagem na formação das escolas ProUCA os formadores solicitaram reflexão em grupo. No entanto, para a criação e execução dos projetos não houve direcionamento de grupos, o que pareceu terem realizado uma aproximação por afinidade pessoal.

\subsection{Projetos desenvolvidos}

Os projetos descritos nesta seção foram desenvolvidos nas escolas ProUCA durante a formação dos professores e gestores nos anos de 2011 e 2012. Foram escolhidos três projetos de escolas diferentes, do ensino fundamental II e do Ensino Médio, são eles:

\section{A) O classmate e a leitura de imagens:}

- Objetivou a leitura crítica de imagens em conteúdos de diferentes áreas do conhecimento.

- Envolveu os professores: espanhol sobre o desmatamento na América Latina; língua portuguesa sobre o reconhecimento da importância do computador na leitura e na escrita para a divulgação de problemas ambientais; geografia no reconhecimento da evolução dos tempos e transformações em imagens naturais e sociais; artes na releitura de imagens sobre o meio ambiente; ciências na busca de imagens de animais com risco de extinção devido ao desmatamento e matemática na construção de gráficos e tabelas sobre o crescimento do desmatamento no Brasil.

- Utilizou os recursos de edição de texto, imagem e apresentação, internet, planilha eletrônica.

- Avaliou a investigação, produção e análise das imagens, a comunicação oral nas exposições e compartilhamento do trabalho.

\section{B) Analfabetismo no bairro:}

- Objetivou identificar o analfabetismo e a escolaridade dos moradores no bairro da escola.

- Envolveu os professores: história elaboração juntamente com os alunos de roteiro de 
pesquisa para as entrevistas em campo com moradores; matemática orientou na construção de tabelas e gráficos e na análise dos dados.

- Utilizou os recursos de edição de texto, planilha eletrônica, postagem em blog.

- Avaliou a tomada de consciência sobre a valorização dos estudos.

\section{C) O materialismo histórico-dialético:}

- Objetivou a apresentação dos principais conceitos da filosofia contemporânea com ênfase no materialismo histórico-dialético.

- Envolveu os professores: artes na exibição de filme e audição de música; filosofia e sociologia com a apresentação dos conceitos filosóficos; matemática na construção de tabelas e gráficos, inglês na tradução de termos encontrados nas leituras e filme.

- Utilizou os recursos da internet, edição de vídeo e som, edição de texto, planilha eletrônica, DVD e aparelho de som.

- Avaliou, por meio da observação, os momentos de investigação e envolvimento dos alunos na execução das atividades.

\subsection{Categorização dos projetos}

Tendo como base as definições e características de experiências multidisciplinares e interdisciplinares expostos na seção 3.1 categorizou-se os projetos A, B e C.

O Projeto A é categorizado como multidisciplinar por apresentar características de disciplinas individualizadas em sua metodologia e forma de pesquisa, apesar de tratar de um tema social para reflexão do grupo, o meio ambiente. O Projeto B é categorizado como multidisciplinar por apresentar características de disciplinas individualizadas também em sua metodologia e forma de pesquisa, apesar de tratar de um tema de interesse dos alunos e relevância histórico-social, totalmente relacionado à realidade local concreta, o analfabetismo no bairro. O Projeto $\mathrm{C}$ é categorizado como multidisciplinar por apresentar características de disciplinas individualizadas novamente em sua metodologia e forma de pesquisa, apesar de haver cooperação e intercâmbio real e prossível enriquecimento mútuo entre os professores, já que o tema estava relacionado a uma única disciplina, a filosofia contemporânea.

Em resumo, os três projetos criados pelos professores e aplicados aos alunos durante a formação do ProUCA apresentam características de experiências multidisciplinares, como relacionados no quadro abaixo (tabela 1 ). 
Tabela 1: Categorização dos projetos do ProUCA em formação de professores

\begin{tabular}{|c|c|c|c|c|}
\hline \multirow{2}{*}{\multicolumn{2}{|c|}{ CARACTERÍSTICAS DAS ABORDAGENS }} & \multicolumn{3}{|c|}{$\begin{array}{c}\text { PROJETOS ESCOLAS } \\
\text { ProUCA }\end{array}$} \\
\hline & & $\mathbf{A}$ & B & $\mathbf{C}$ \\
\hline \multirow{3}{*}{ 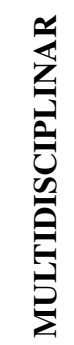 } & Solução de problema específico & & & \\
\hline & Diversidade de metodologia & & & \\
\hline & Pesquisa disciplinar & & & \\
\hline \multirow{4}{*}{ 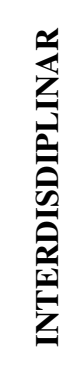 } & $\begin{array}{l}\text { Solução de problema específico } \\
\text { relacionado à realidade }\end{array}$ & & & \\
\hline & Compartilhamento de metodologia & & & \\
\hline & Fusão entre campos & & & \\
\hline & Coordenação integradora & & & \\
\hline
\end{tabular}

\section{Reflexões sob os pressupostos do m-learning}

Como parte integrante de um programa voltado ao m-learning, os projetos desenvolvidos pelas escolas do ProUCA aqui descritos, não demonstraram irem muito além da cooperação entre professores. Tendo em vista as características do m-learning, podemos ressaltar algumas reflexões.

- Interação entre professores/alunos e professores/professores: os professores demonstraram, no desenvolvimento de seus projetos ( $\mathrm{A}, \mathrm{B}$ ou $\mathrm{C}$ ), que ainda não interagem com seus alunos como autônomos capazes de criação e análise, já que as avaliações eram baseadas no desempenho do aluno na realização das atividades propostas. Pode-se verificar grande interação entre professores, principalmente, no que diz respeito a utilização das tecnologias, em especial o laptop UCA.

- Contribuição, participação e acesso ao ambiente de aprendizagem em outros formatos: observa-se o uso da metodologia mais centrada no professor que ensina. Não se pode inferir uso de agrupamentos de alunos e tampouco se foi observado o seu conhecimento prévio para o desenvolvimento dos projetos. Houve no Projeto B uma mudança de ambiente com a pesquisa em campo, porém não ficou claro se foram utilizados os laptops UCA para tal. E o Projeto C fez uso de outras tecnologias existentes na escola. 
- Aspectos técnicos: os recursos de edição de texto, Internet e planilha do laptop foram utilizados nos três projetos, demonstrando a importância destes recursos no uso educacional do equipamento. Já o editor de imagens e som, câmera de foto e vídeo e editor de apresentação também foram utilizados.

- Contribuição para administração e gestão: nos três projetos não se observou contribuição específica para e da administração e gestão nas questões tecnológicas, mas com certeza houve incentivo para a realização dos mesmos.

Portanto, pode-se resumir (Tabela 2):

Tabela 2: Resumo reflexões dos projetos multidisciplinares no $m$-learning

\begin{tabular}{|c|c|}
\hline Pressupostos m-learning & Projetos multidisciplinares \\
\hline $\begin{array}{l}\text { Interação entre professores e } \\
\text { alunos, interação entre professores }\end{array}$ & $\begin{array}{l}\text { - Interação com alunos sem autonomia, criação e } \\
\text { possibilidade de análise crítica; } \\
\text { - Avaliações voltadas unicamente ao desempenho dos } \\
\text { alunos nas atividades propostas; } \\
\text { - Cooperação entre professores, sem grandes mudanças } \\
\text { do seu próprio fazer. }\end{array}$ \\
\hline $\begin{array}{l}\text { Contribuição, participação e } \\
\text { acesso ao ambiente de } \\
\text { aprendizagem em outros formatos }\end{array}$ & $\begin{array}{l}\text { - Metodologia centrada no professor; } \\
\text { - Pouca mudança de ambiente, não se demonstrou a } \\
\text { mobilidade dos laptops UCA como possibilidade de } \\
\text { avanço pedagógico e didático; } \\
\text { - Combinação com outras tecnologias da escola. }\end{array}$ \\
\hline Aspectos técnicos & $\begin{array}{l}\text { - Grande utilização do editor de texto, Internet e } \\
\text { planilha; } \\
\text { - Utilização também do editor de imagens, som, } \\
\text { apresentação, câmera de foto e vídeo. }\end{array}$ \\
\hline $\begin{array}{l}\text { Contribuição para e da gestão e } \\
\text { administração }\end{array}$ & - Certeza de incentivo à efetivação dos projetos \\
\hline
\end{tabular}

\section{Considerações Finais}

As tecnologias móveis educacionais disponibilizadas hoje nas escolas públicas são mais uma possibilidade de oportunizar a inclusão digital e social dos alunos e seus familiares. Um momento histórico imperdível, que merece continuidade, não unicamente para a melhoria da qualidade educacional.

Uma instituição de aprendizagem, que se organiza para o uso destas TIC, que procura promover transformações nas concepções metodológicas, que busca criar novas ações pedagógicas 
que provoque mudanças curriculares, que possibilita, por meio de um trabalho colaborativo, a participação ativa do aluno, para que este desenvolva sua autonomia e seja protagonista da própria aprendizagem, demonstra o empenho da comunidade escolar. Acredita-se, desta forma, estar no caminho de um ambiente de aprendizagem móvel voltado para a real melhoria da qualidade educativa.

A criação e desenvolvimento de Projetos de Aprendizagem multidisciplinares ou interdisciplinares de forma reflexiva apresenta uma mudança significativa nos espaços e tempos da escola, na metodologia de ensino e no desempenho final dos alunos, como sujeitos críticos, inclusive no papel da escola na sociedade.

Para tal, também é preciso que os projetos voltados para aprendizagem móvel verifiquem as possibilidades físicas e técnicas de cada espaço a ser implantado, que utilizem tecnologias desenvolvidas dentro de estudos de usabilidade e os atributos da computação ubíqua, que também busquem soluções para questões administrativas e de gestão pedagógica e, por fim, que promovam formação contínua e permanente, que respeite o caminhar dos seus agentes - professores, gestores e alunos, assim como os projetos políticos pedagógicos das escolas.

\section{Referências Bibliográficas}

Anais do I Congresso Brasileiro de Informática na Educação - CBIE, Rio de Janeiro, 2012. Disponível em http://www.cbie.org.br/.

Anais do Simpósio Brasileiro de Informática na Educação - SBIE, Aracaju, SE, 2011. Disponível em http://www.br-ie.org/index.php/anaissbie.

BICALHO, L.Ma; OLIVEIRA, M. "Aspectos conceituais da multidisciplinaridade e da interdisciplinaridade e a pesquisa em ciência da informação". Revista Eletrônica de Biblioteconomia e Ciência da Informação, v. 16, n. 32, p. 1-26, 2011.

ALMEIDA, M. E. B. e PRADO, M. E. B. B. "Indicadores para a formação de educadores para a integração do laptop na escola". In Almeida, M. E. B.; Prado, M. E. B. B. (Org.) O computador portátil na escola: mudanças e desafios nos processos de ensino e aprendizagem. São Paulo: Avercamp, 2011.

BONA, A.S.; MORAIS, A.D.; MATTOS, E.B. V.; ROSA, M. B.; BASSO, M.V. A.; FAGUNDES, L. "Concepções de Currículo, Projetos de Aprendizagem e Interação no Projeto UCA/RS". Anais do XXII SBIE - XVII WIE Aracaju, 21 a 25 de novembro de 2011.

BRASIL. Um computador por aluno: a experiência brasileira. Brasília: Câmara dos Deputados, Coordenação de Publicações, 2008.

BRASIL. "UCA: Um Computador por Aluno. O Projeto. Quando Começou". Ministério da Educação. Disponível http://www.uca.gov.br/institucional/. Acesso em 31/01/2013.

DANNEMANN, A.C. "O desafio do uso da tecnologia na prática de sala de aula" in Pesquisa sobre o uso das tecnologias de informação e comunicação no Brasil: TIC Educação 2012. São Paulo. Comitê Gestor da Internet no Brasil, 2013.

DERIQUITO, M.; DOMINGO, Z. MOBILE LEARNING FOR TEACHERS: "Exploring the Potential of Mobile Technologies to Support Teachers and Improve Practice". Working Paper 
Series on Mobile Learning. UNESCO, Paris, France, 2012.

FAGUNDES, L. da C.; JOST, D.F. "Que contribuições podemos buscar na experiência piloto 'Projeto UCA' proposto pelo MEC no Brasil em 2010-2012?" in Pesquisa sobre o uso das tecnologias de informação e comunicação no Brasil: TIC Educação 2012. São Paulo. Comitê Gestor da Internet no Brasil, 2013.

FREITAS D. Filho, N.; BARBOSA, E. F. "Estudo e Definição de um Conjunto de Características e Requisitos para Ambientes de Aprendizagem Móvel". Anais I Congresso Brasileiro de Informática na Educação-CBIE, RJ, 2012.

FRIGOTTO, G. "A Interdisciplinaridade como necessidade e como problema nas ciências sociais". In: BIANCHETTI. L. , JANTSCH. A. Interdisciplinaridade: para além da filosofia do sujeito. Petrópolis: Vozes. 1995. p. 20- 62.

ISAACS, S. "Turning On Mobile Learning In Africa And The Middle East". Working Paper Series on Mobile Learning. UNESCO,Paris, France, 2012.

MUNIZ, R. de F.; MUNIZ, S.M.; Lima, L.L.V.; SILVA, M.A.; CASTRO FILHO, J.A. "Raízes do Nordeste: a experiência do uso dos laptops educacionais no trabalho com projeto multidisciplinar". WUCA. CBIE, Rio de Janeiro, 2012.

NASCIMENTO. A.C.T.A.A. “A integração das tecnologias à práticas escolares” in Pesquisa sobre o uso das tecnologias de informação e comunicação no Brasil: TIC Educação 2012. São Paulo. Comitê Gestor da Internet no Brasil, 2013.

NOGUEIRA, N. R. "Pedagogia dos projetos: uma jornada Interdisciplinar rumo ao desenvolvimento das múltiplas inteligências”. São Paulo. Érica, 2001.

OLPC. Disponível em http://one.laptop.org/, acesso em 31/01/2013.

SANTOS, M. B. F.; BORGES, M.K. “O Uso de Laptops Na Escola: Alterações e Permanência no Currículo". Anais do XXII SBIE - XVII WIE Aracaju, 2011.

SILVA, C. V. A. P.; PETRUCCI, M. R. "Formação de educadores em programas de educação mediada por tecnologias digitais: experiências no estado da Paraíba". I Congresso Brasileiro de Recursos Digitais na Educação. Mackenzie, São Paulo, 2012.

UNESCO. Policy Guidelines for Mobile Learning (Version 2.1: Draft), 2012.

VENANCIO, V.; CORREA, A.G.D; TELLES, E.O, CORREA, C.M; GARCIA, B.V.R; RIBEIRO, C.C; FICHEMAN, I.K; LOPES, R.D. "Integração dos Laptops Educacionais ao Cotidiano Escolar no UCA São Paulo: facilidades e dificuldades". I Congresso Brasileiro de Recursos Digitais na Educação. Mackenzie, São Paulo, 2012a.

VENANCIO, V. "Forma e metodologia de trabalho no uso de tecnologias móveis educacionais dentro da formação UCA: relato de experiência em formação presencial". WUCA. CBIE, Rio de Janeiro, 2012b.

VENANCIO, V.; FICHEMAN, I.K.; LOPES, R.D.. "Reflexões sobre avaliação da formação local de educadores do ProUCA sob os pressupostos do m-learning". II Congresso Brasileiro de Recursos Digitais na Educação. Mackenzie, São Paulo, 2013. 